\title{
Análisis del comportamiento de flujo de fluidos en un horno de reverbero agitado con diferentes impulsores, mediante la modelación física y numérica ${ }^{(*)}$
}

\author{
R. Juárez*, A. Flores*, E. Macias** y N. Reyes*
}

\begin{abstract}
Resumen
Utilizando la simulación física y numérica, en este trabajo se presenta un análisis del flujo de fluidos y la trayectoria de partículas, en un modelo escala 1:2 de un horno de reverbero de 5 t. La caracterización del sistema sirvió para determinar el comportamiento del líquido en un sistema agitado mediante impulsores de diferentes formas rotando a diferentes velocidades y alturas, lo cual ha permitido determinar tiempos de circulación, de mezclado, patrones de flujo y patrones de velocidad en las cubas de agitación y fusión. Además, se aportan datos experimentales de la interacción entre flujos turbulentos y partículas sólidas. Los resultados obtenidos sugirieron cambios en la geometría del diseño de un horno convencional, propiciando mezclado más rápido y eficiente e incrementando la eficiencia en la remoción de magnesio.
\end{abstract}

\section{Analysis of fluid flow in a stirred reverberatory furnace with different impellers, by physical and numerical modeling}

Abstract

Keywords

\begin{abstract}
A fluid flow analysis and particle trajectory in a $5 \mathrm{t}$ molten capacity scaled 1:2 model of a reverberatory furnace was performed using both physical and numerical simulation. The design of the system allowed studying the behavior of the liquid in a system agitated by impellers. The parameters studied were, rotating speed, shape and height of the impeller. The physical and numerical simulations were used to determine circulation and mixing times, fluid flow patterns and velocity profiles in the agitation and melting chambers. Using experimental data, it was possible to establish the interaction between turbulent flow and circulating solid particles. The original design of the reverberatory furnace was modified based on the physical and numerical simulation analysis. The new design showed an increased magnesium removal rate by means of a more efficient and faster mixing.
\end{abstract}

Aluminum refining; Turbulent multiphase flow; Mathematical modeling; Physical modeling.

\section{INTRODUCCIÓN}

La agitación en reactores se usa, ampliamente, en procesos industriales para favorecer el mezclado de fluidos individuales o multifase con otros líquidos o sólidos. A su vez, el mezclado es una operación unitaria que influye sobre el consumo de energía y la operación efectiva de procesos industriales, debido a su gran influencia sobre fenómenos de reacción que tienen lugar durante los mismos. Por lo tanto, las operaciones de mezclado comprenden una parte substancial del costo total de la manufactura de artículos en industrias como la metalúrgica, la química, la agrícola, etc. Uno de los parámetros cruciales es el tiempo de mezclado, definido como el tiempo que tardan en unirse eficientemente los reactantes agregados en un reactor ya que, por ejemplo, en hornos de reverbero usados para el reciclaje y refinación de aluminio secundario, este influye sobre el gasto de combustible y la operación eficiente del horno ${ }^{[1]}$.

Yeoh $^{[2]}$ realizó un trabajo sobre el tiempo de mezclado y el grado de homogeneidad en reactores usados en la industria farmacéutica, empleando una cámara estándar agitada por un impulsor Rushton.

(•) Trabajo recibido el día 19 de enero de 2009 y aceptado en su forma final el día 13 de abril de 2009

* Centro de Investigación y Estudios Avanzados del IPN, Unidad Saltillo, Méjico.

** Universidad Autónoma de San Luis de Potosí, Méjico. 
Describió que a través de la cámara existen diferentes zonas de mezclado y flujo turbulento por encima y por debajo de las aspas del impulsor. Investigaciones recientes ${ }^{[3}{ }^{4]}$ han establecido que dentro de los reactores agitados existen diferentes clases de inestabilidades, las cuales se presentan como fluctuaciones de flujo, espaciadas y permanentes. Las inestabilidades pueden tener implicaciones benéficas sobre el proceso de mezclado y sobre la eficiencia del movimiento del fluido para alcanzar la homogeneización a través de variaciones de flujo. De igual modo, las inestabilidades tienen efectos similares como aquellos publicados por Murakami et al..$^{[5]}$ y Nomura et al. ${ }^{[6]}$ para el mezclado en tanques agitados. Yao et al. ${ }^{[7]} \mathrm{pu}-$ blicaron que existen zonas de mezclado aislado, por encima de las aspas del impulsor y por debajo de éste. En sus resultados experimentales, muestran que el tiempo de mezclado se reduce significativamente al aplicar un cambio en el sentido de agitación. Barresi ${ }^{[8]}$ investigó la interacción entre el flujo turbulento y reacciones que ocurren rápidamente y concluyó que la velocidad de reacción se ve afectada por variables tales como grado de agitación, temperatura del baño, tamaño de partícula, tiempo de residencia de las partículas, etc. Una aplicación de estas interacciones se ha dado durante el estudio de la eficiencia de la reacción entre magnesio y sílice para la refinación de aluminio secundario a nivel laboratorio ${ }^{[9]}$. Un factor de gran importancia es el tiempo de residencia de las partículas inyectadas, el cual se ha medido para la reacción de magnesio con partículas de sílice. Se ha encontrado que este es muy corto, inferior a $3 \mathrm{~s}$, pero suficiente para que la reacción se lleve a cabo eficientemente ${ }^{[10]}$. Sin embargo, también se ha investigado que, bajo condiciones de pobre agitación, los productos de reacción permanecen adheridos a las partículas agregadas, restringiendo la difusión del magnesio para seguir reaccionando con el núcleo de sílice. En consecuencia, se ha determinado la aparición de gran cantidad de inclusiones no metálicas en el producto solidificado[11].

Con base en lo anterior, el objetivo de este trabajo es caracterizar el comportamiento del flujo de fluidos dentro de un horno de reverbero agitado con impulsores de diversas geometrías, para determinar los tiempos de circulación, los tiempos de mezclado y la trayectoria de partículas, mediante el modelado físico y numérico. Los patrones de flujo obtenidos del modelo físico se utilizaran para describir los patrones de flujo en el horno de reverbero agitado a velocidad constante, para, posteriormente, compararlos con los obtenidos en la etapa de simulación numérica. Esto último, se ha llevado a cabo mediante el software FLUENT, utilizando el modelo $K-\varepsilon^{[12}$ y 13$]$ para determinar los campos de velocidad del fluido en el sistema y las trayectorias de partículas.

\section{DESARROLLO EXPERIMENTAL}

\subsection{Modelo físico simple}

Esta etapa del desarrollo experimental se realizó en un modelo físico, escala (1:2), de un horno de reverbero (prototipo). El modelo físico y los impulsores se ilustran en las figuras 1 ( $\mathrm{a}$ y b)), donde el impulsor turbina se define como IT, mientras que los impulsores de dos y tres paletas como I2P e I3P, respectivamente. Las dimensiones del modelo y de los impulsores se presentan en la tabla I. Las propiedades físicas del fluido de trabajo (agua) se presentan a continuación: la densidad tiene un valor de $1.000 \mathrm{~kg} / \mathrm{m}^{3}$, con una viscosidad de $8,9031 \times 10^{4} \mathrm{~kg} / \mathrm{m} \cdot \mathrm{s}$, las viscosidades dinámica y cinemática fueron de $1 \times 10^{-3} \mathrm{~kg} / \mathrm{m} \cdot \mathrm{s}$ y $1 \times 10^{-7} \mathrm{~m}^{2} / \mathrm{s}$, respectivamente, con una tensión superficial de 0,0072 $\mathrm{N} / \mathrm{m}^{-1}$. En este prototipo se preparan aleaciones de aluminio (A319, A380), las cuales, generalmente a $660^{\circ} \mathrm{C}$, presentan una densidad de $2.368 \mathrm{~kg} / \mathrm{m}^{3}$, una viscosidad dinámica de $1,25 \times 10^{-3} \mathrm{~kg} / \mathrm{m} \cdot \mathrm{s}$, una viscosidad cinemática de $5,30 \times 10^{-7} \mathrm{~m}^{2} / \mathrm{s}$ y una tensión superficial de $0,0868 \mathrm{~N} / \mathrm{m}^{-1}$.

En el presente estudio se evaluaron los efectos de los siguientes factores sobre los tiempos de circulación y de mezclado: relación de alturas, hi/h (altura del impulsor respecto al fondo de la cuba), $T$ es el tipo del impulsor, $v$ es la del impulsor. El desarrollo de las pruebas en el modelo físico se realizó según las condiciones experimentales dinámicas y geométricas cuyos valores aparecen en la tabla II, donde I es el tipo de impulsor, $h i / h$ es la relación de altura del

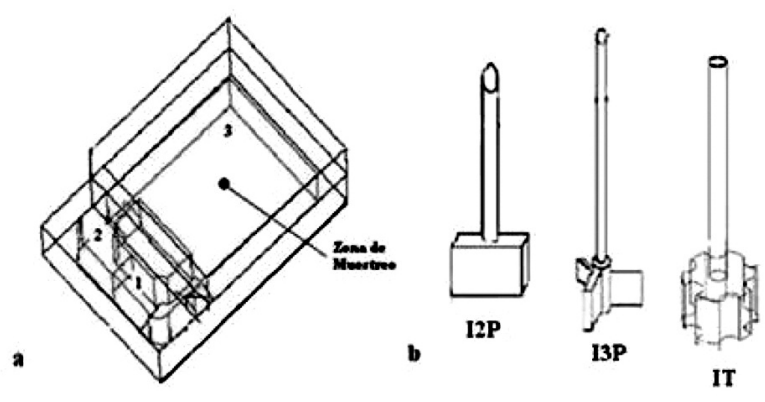

Figura 1. a) Esquema del modelo físico del horno de reverbero, escala (1:2); b) Impulsores utilizados. Las dimensiones de cada cuba y de las paletas de los impulsores se especifican en la tabla I.

Figure 1. a) Scheme of the physical model of a reverberatory furnace 5 ton molten aluminum capacity, scale (1:2); b) Impellers utilized. The dimensions of each chamber as well as of the blades of each impeller are given in table I. 
Tabla I. Dimensiones de cada cuba en el modelo físico y de las paletas de los impulsores utilizados

Table I. Dimensions of each one of the chambers in the physical model and of the blades of the impellers utilized

\begin{tabular}{ccccc}
\hline Cuba & Altura & Diámetro & Ancho & Largo \\
\hline $\begin{array}{l}\text { Agitación } \\
\text { Tratamiento } \\
\text { Fusión }\end{array}$ & $\begin{array}{c}42,5 \\
42,5\end{array}$ & 50 & - & - \\
\hline Impulsor & 42,5 & 50 & 70 & 80 \\
& Ancho del & Diámetro del & Largo del & aspa \\
impulsor & $\begin{array}{c}\text { Altura del } \\
\text { aspa }\end{array}$ \\
\hline$I T$ & 2,5 & 9,5 & 2,5 & 7,5 \\
$I 3 P$ & 3,8 & 17,5 & 8,5 & 8 \\
\hline
\end{tabular}

* Todas las dimensiones están dadas en cm.

Tabla II. Condiciones experimentales dinámicas y geométricas empleadas

Table II. Experimental geometrical and dynamic conditions studied

\begin{tabular}{cccccc}
\hline \multicolumn{2}{c}{ Condición Experimental } & \multicolumn{2}{c}{$\begin{array}{c}\text { Variables de } \\
\text { respuesta }\end{array}$} \\
\cline { 1 - 4 } Dinámica & \multicolumn{2}{c}{ Geométrica } & & \\
\hline $\boldsymbol{N}$ (rpm) & $\boldsymbol{I}$ & $\boldsymbol{h i} / \boldsymbol{h}$ & $\boldsymbol{D} / \boldsymbol{T}$ & $\boldsymbol{t}_{\boldsymbol{c}}(\mathbf{s})$ & $\boldsymbol{t}_{\boldsymbol{m}}(\mathbf{s})$ \\
\hline 200 & $I 2 P$ & 0,21 & 0,72 & 24 & 120 \\
200 & $I 2 P$ & 0,32 & 0,72 & 32 & 120 \\
200 & $I T$ & 0,12 & 0,34 & 30 & 60 \\
200 & $I T$ & 0,18 & 0,34 & 30 & 110 \\
351 & $I T$ & 0,12 & 0,34 & 30 & 60 \\
351 & $I T$ & 0,18 & 0,34 & 31 & 50 \\
255 & $I 3 P$ & 0,25 & 0,71 & 21 & 84 \\
255 & $I 3 P$ & 0,30 & 0,80 & 15 & 96 \\
292 & $I 3 P$ & 0,25 & 0,71 & 14,5 & 72 \\
292 & $I 3 P$ & 0,30 & 0,80 & 15 & 84 \\
\hline
\end{tabular}

impulsor, $D / T$ es la relación de diámetro entre el impulsor y la cuba, $t_{c}$ corresponde al tiempo en que el colorante recorre todo el sistema. También, se determinó el tiempo de mezclado, que se define como el período en que se alcanza una concentración constante del colorante en el sistema. La secuencia de una prueba típica se describe a continuación: el modelo físico fue llenado con agua (160 o 180 1). Una vez lleno el modelo, se inicia la rotación del impulsor a la velocidad de estudio; después de 10 s de rotación se añaden $50 \mathrm{~g}$ de un colorante, en un solo evento y en el mismo lugar. Para asegurar la velocidad constante del impulsor se utiliza un motor controlado por microprocesadores. El muestreo se realiza en la cuba de fusión, en la zona del centro de la misma, a una profundidad de $9 \mathrm{~cm}$ desde el fondo de la cuba, en el punto marcado en la figura 1 . Se eligió este punto de muestreo debido a estas tres situaciones: su forma geométrica, ya que es donde se encuentra la mayor parte de líquido; que se tiene acceso directo al seno del líquido; y que es la zona donde se requiere saber si la refinación se ha llevado a cabo.

El análisis colorimétrico se realizó en un espectrofotómetro Shimadzu, modelo UV-1201, donde los valores de coloración se expresan en términos de absorbancia, (A). En este trabajo se consideró que la determinación de tiempos de mezclado era más confiable usando determinaciones mediante espectrometría de luz ultravioleta, radioactiva, química, fluorescente o electrónica ${ }^{[14]}$. Finalmente, la verificación experimental del comportamiento líquido-partícula en el modelo físico se realizó mediante la adición de partículas de baquelita al modelo, siguiendo el programa de pruebas mostrado en la tabla II.

Para la construcción y desarrollo de pruebas en el modelo físico se tomaron en cuenta criterios de similitud geométricos y mecánicos. A su vez, la similitud mecánica se subdividió en criterios de similitud cinemática y dinámica entre un modelo y su prototipo, siendo la dinámica la de mayor interés, en este caso. 
Los criterios que asemejan mejor las condiciones del prototipo son el del número de Reynolds, cuyo valor permite determinar cuándo se pasa de un régimen laminar a uno turbulento, y el Número de Froude, que representa la relación entre la fuerza de inercia causada por el impulsor y la fuerza de gravedad que se le opone. En el presente caso, el valor de $N_{m}=1,414 \mathrm{Mp}$ y el cual se calculó utilizando la siguiente expresión:

$$
N_{m}=N_{p}\left(\frac{D_{P}}{D_{m}}\right)^{1 / 2}
$$

Los valores de los criterios de similitud que se calcularon y las condiciones experimentales se presentan en forma resumida en la tabla III, donde $\mathrm{Nm}$ corresponde a la velocidad de rotación en el modelo y $\mathrm{Np}$ a la del prototipo industrial. Una presentación más amplia respecto a los procedimientos analíticos para determinar los valores de los parámetros descritos en dicha tabla puede ser encontrada en un trabajo incluido en la bibliografía ${ }^{[15]}$.

\subsection{Modelo físico con modificaciones}

Como se discutirá más adelante, durante la primera etapa experimental, se determinaron tiempos de circulación y mezclado relativamente largos, así como la presencia de zonas estancadas en algunas partes de la cuba fusión, por lo que se creyó conveniente realizar algunas modificaciones geométricas a la cuba con el fin de mejorar las condiciones de mezclado. Las modificaciones consistieron en poner dos trampas donde el líquido presenta las velocidades más bajas, es decir, a la mitad de la longitud de la cuba. Dichas trampas tienen un ancho de $15 \mathrm{~cm}$ y una altura de $25 \mathrm{~cm}$, con un cuerpo acoplado tipo paleta posicionado de frente a la dirección de flujo para ayudar a romper el patrón de flujo que se presenta e incrementar la velocidad del líquido. También, se modificó la parte posterior de la cuba, reduciendo las esquinas, ya que en estas se forman zonas estancadas. Dichas esquinas se redondearon hacia adentro con el fin de que el líquido que alcance esta parte de la cuba tenga ayuda por parte de la pared para no reducir la velocidad que este lleva. En la tabla IV se muestran las condiciones bajo las que se realizaron las pruebas para esta nueva situación. Cabe mencionar que estas son las condiciones que presentaron mejores resultados a lo largo del estudio. Si se comparan los tiempos de circulación y mezclado de las tablas II y IV se puede ver que las modificaciones tienen un efecto benéfico, ya que los tiempos de circulación y mezclado se han reducido en muchos casos.

\subsection{Modelación numérica}

Las simulaciones se llevaron acabo de acuerdo al desarrollo experimental del modelo físico, donde se aplica el modelo $K-\varepsilon$, el cual se hautilizado para

Tabla IV. Condiciones experimentales en el modelo modificado

Table IV. Set of experimental conditions for the modified physical model

\begin{tabular}{|c|c|c|c|c|c|}
\hline \multicolumn{4}{|c|}{ Condición Experimental } & \multirow{2}{*}{\multicolumn{2}{|c|}{$\begin{array}{l}\text { Variables de } \\
\text { respuesta }\end{array}$}} \\
\hline \multicolumn{2}{|c|}{ Dinámica } & \multicolumn{2}{|c|}{ Geométrica } & & \\
\hline$N(r p m)$ & I & $h i / h$ & $D / T$ & $t_{c}(s)$ & $t_{m}(s)$ \\
\hline 200 & $I T$ & 0,12 & 0,12 & 23 & 53 \\
\hline 200 & $I T$ & 0,18 & 0,18 & 28 & 89 \\
\hline 351 & $I T$ & 0,12 & 0,12 & 17 & 36 \\
\hline 351 & $I T$ & 0,18 & 0,18 & 27 & 44 \\
\hline 255 & $13 P$ & 0,25 & 0,25 & 14 & 36 \\
\hline 255 & $13 P$ & 0,30 & 0,30 & 12 & 48 \\
\hline 292 & $13 P$ & 0,25 & 0,25 & 12 & 48 \\
\hline 292 & $13 P$ & 0,30 & 0,30 & 13 & 60 \\
\hline
\end{tabular}

Tabla III. Velocidades de rotación solicitadas en el modelo físico, según los criterios de similitud dinámica contemplados

Table III. Rotation velocities solicited in the physical model, according to the similarity dynamic criterions evaluated

\begin{tabular}{lccc}
\hline \multicolumn{1}{c}{ Criterio } & Relación entre $\mathbf{N m}$ y $\mathbf{N p}$ & $\mathbf{N p}(\mathbf{r p m})$ & $\mathbf{N m}(\mathbf{r p m})$ \\
\hline Numero de Reynolds & $N m=7.584 N p$ & 180 & 1365 \\
Numero de Froude & $N m=1.414 N p$ & 180 & 255 \\
\hline
\end{tabular}


investigar patrones de flujo en tanques agitados mediante un impulsor. En primer lugar, se procedió a realizar las simulaciones de la cuba de agitación, ya que en esta es donde se encuentra el impulsor y es la zona que proporciona fuerza al líquido para moverse por las secciones restantes hasta completar la circulación. Después, se simuló el flujo en la cuba de fusión, donde para realizar estas simulaciones se tomaron en cuenta los valores experimentales arrojados por el modelado físico, tales como tiempo de circulación, tiempo de mezclado y la velocidad promedio del líquido. Para el desarrollo de las simulaciones en la cuba de agitación se emplearon coordenadas cilíndricas, mientras que en el caso de la cuba de fusión se utilizaron coordenadas rectangulares. Para desarrollar la malla, introducir las ecuaciones del modelo $K-\varepsilon$, las condiciones a la frontera requeridas y los valores de las propiedades de fluido se utiliza el software FLUENT, que se utiliza, ampliamente, para el análisis del flujo de fluidos en sistemas como el que se desea simular. De esta forma se obtienen patrones de flujo, perfiles de velocidad, patrones de presión, trayectorias de partículas, etc., que caracterizan el comportamiento hidrodinámico del sistema.

\section{RESULTADOS Y DISCUSIÓN}

\subsection{Modelo físico simple}

En este estudio, el comportamiento en la cuba de agitación es completamente turbulento (para los impulsores usados) de acuerdo a los valores de los números de Reynolds para tanques agitados medidos en la zona, como se muestra en la tabla V. El número de Reynolds es definido usualmente para tanques agitados como:

$$
\operatorname{Re}=\frac{\rho N_{r p s} D_{i}^{2}}{\mu}
$$

donde $N_{r p s}$ es la velocidad del impulsor en revoluciones por segundo, $D_{i}$ es el diámetro del impulsor, $\rho$ es la densidad del fluido, $\mu$ es la viscosidad del fluido. El número de Reynolds es un parámetro importante que describe si las condiciones de flujo conducen a un flujo laminar o turbulento. En flujos laminares, el número de Reynolds es menor que $N_{\mathrm{Re}}<10.000$, el cual es el valor límite para tanques agitados.

La velocidad del fluido varía significativamente en las cubas de tratamiento y agitación, comparada con la cuba de fusión. Esto se explica mejor observando la figura 2 , que indica la velocidad medida a la entrada de cada una de las compuertas. El líquido a la entrada de la cuba de tratamiento tiene una velocidad lineal de $14 \mathrm{~cm} / \mathrm{s}$. La velocidad se reduce aproximadamente $6 \mathrm{~cm} / \mathrm{s}$ a la entrada de la cuba de fusión. Ahora, el régimen del líquido ha cambiado de turbulento a laminar. El lento movimiento del líquido en la cuba de fusión permanece constante durante todo el ciclo de mezclado, incluso aunque la cuba de agitación aún produce energía y "momentum". También, en esta figura se puede observar que la alta velocidad del fluido fue para la relación $h i / h=$ 0,12 , donde las aspas del impulsor están localizadas justo en el centro de la compuerta, esperando que no interaccione el fluido con las paredes de la cuba.

La comparación del flujo de fluido par los diferentes impulsores bajo estudio se presenta a continuación, donde se reportan las mediciones cuantitativas de los tiempos de mezclado. En las figuras 3 y 4 se muestran las graficas correspondientes a la absorbancia contra tiempo para los diferentes tipos de impulsores rotando a diferentes velocidades. En

Tabla V. Números de Reynolds calculados para los impulsores usados en este trabajo y los que se usan a nivel industrial

Table V. Reynolds numbers for the impellers used in this work, and the ones used at industrial level

\begin{tabular}{cccccc}
\hline $\begin{array}{c}\text { Velocidad en } \\
\text { el modelo } \\
\text { (rpm) }\end{array}$ & Impulsor & $\begin{array}{c}\text { Numero de } \\
\text { Reynolds }\end{array}$ & $\begin{array}{c}\text { Velocidad en } \\
\text { el prototipo } \\
\text { (rpm) }\end{array}$ & Impulsor & $\begin{array}{c}\text { Numero de } \\
\text { Reynolds }\end{array}$ \\
\hline 200 & $I 2 P$ & 33451,83 & 164 & $12 P$ & 6539700159,28 \\
255 & $I 2 P$ & 43081,90 & 180 & $13 P$ & 7186483691,52 \\
292 & $I 2 P$ & 48657,20 & 180 & I3P & 7186483691,52 \\
200 & $I T$ & 34060,04 & $*$ & $*$ & $*$ \\
351 & $I T$ & 38520,29 & $*$ & $*$ & $*$ \\
\hline
\end{tabular}

*(Nota: el impulsor IT no se usa a nivel industrial). 


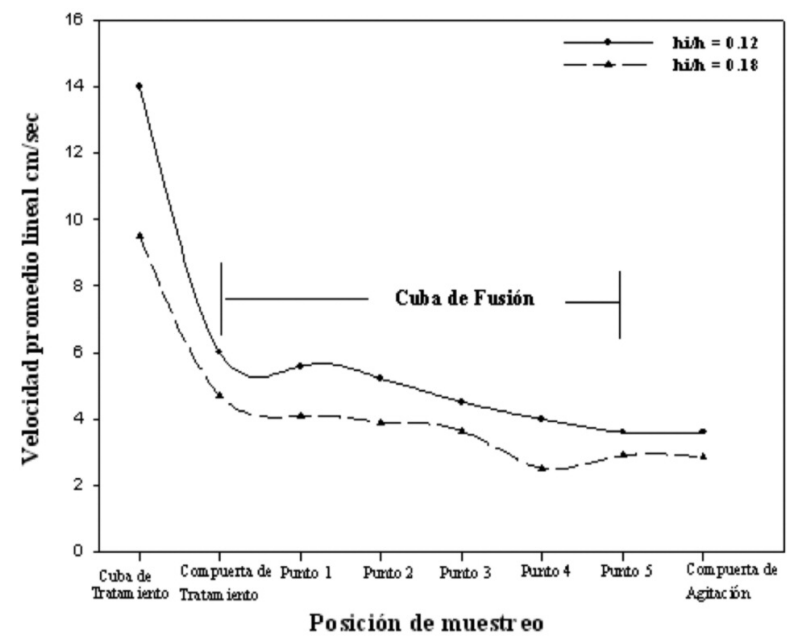

Figura 2. Comportamiento de la velocidad del líquido con relación a la posición de muestreo, para un IT rotando a $200 \mathrm{rpm}$, para las condiciones geométricas indicadas.

Figure 2. Velocity measurements as a function of sampling position in the physical model, for an IT impeller rotating at $200 \mathrm{rpm}$, for the indicated geometrical features.

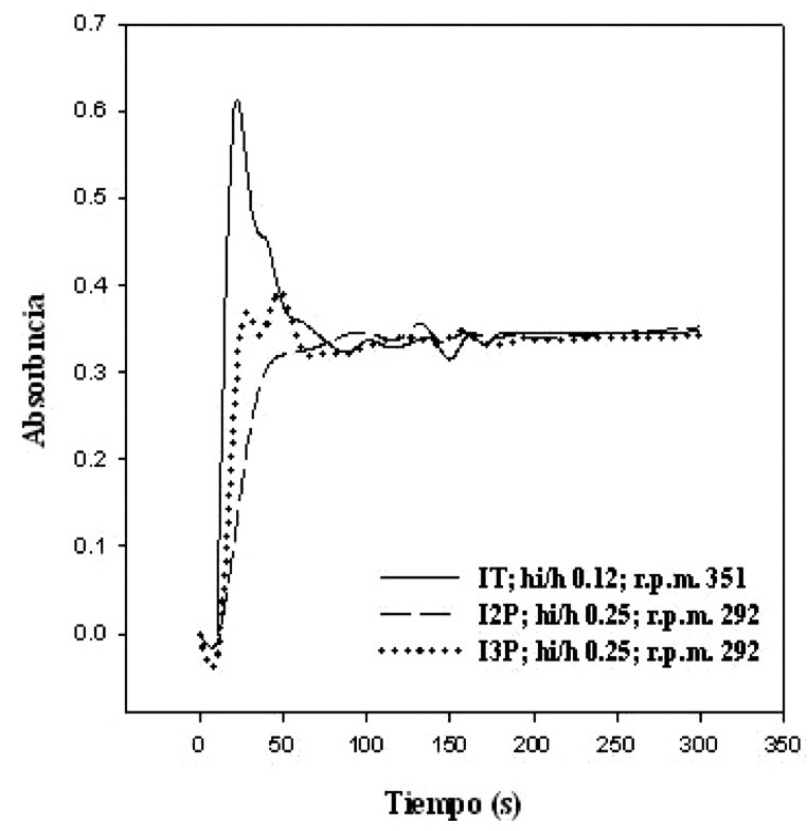

Figura 3. Relación entre la absorbancia y el tiempo de mezclado para diferentes impulsores, para las relaciones $h i / h$ y velocidades de rotación indicadas.

Figure 3. Relationship between absorbance and mixing time for the different impellers, for the indicated hi / $\mathrm{h}$ ratios and rotation speeds.

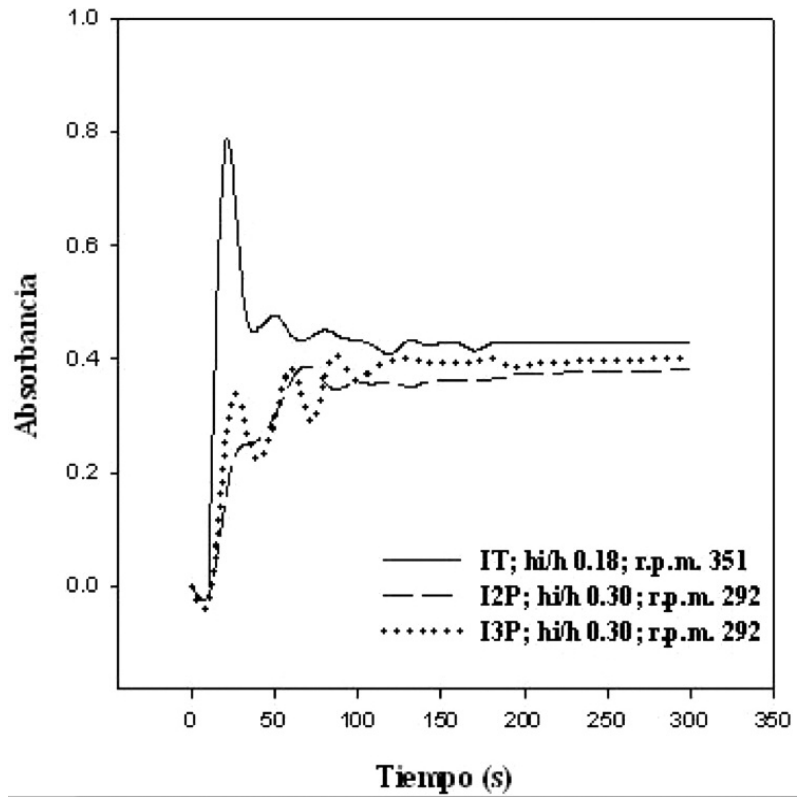

Figura 4. Relación entre la absorbancia y el tiempo de mezclado para diferentes impulsores, para las relaciones $h i / h$ y velocidades de rotación indicadas.

Figure 4. Relationship between absorbance and mixing time for the different impellers, for the indicated hi / $\mathrm{h}$ ratios and rotation speeds.

estas figuras, al principio del mezclado, se observan algunas perturbaciones asociadas al avance del frente de colorante y a la formación de zonas estancadas.

De acuerdo a los resultados que se muestran en las figuras 3 y 4 , se puede observar un mejor funcionamiento de los impulsores tipo paleta, homogenizando los valores de absorbancia en periodos de tiempo más cortos. Sin embargo, se observó la formación de zonas estancadas en la cuba de fusión, caracterizada por la pobre interacción del fluido con sus alrededores, debido al diseño del horno de reverbero. La representación esquemática del comportamiento del flujo de fluido se muestra en una vista superior del modelo físico, en la figura 5, donde se puede apreciar el perfil de flujo, independientemente del tipo de impulsor que se use. Como se observa, el flujo es transportado, siempre a través, por las paredes del modelo hasta la parte posterior, donde las zonas estancadas se forman en las esquinas. El líquido sigue esta ruta, incorporando lentamente "momentum" al resto del sistema, como las fuerzas de inercia en esta parte del modelo permiten que el líquido alcance la compuerta de la cuba de agitación una vez más, completando la recirculación. No obstante, una amplia zona estancada se localiza entre las cubas de tratamiento y agitación, dentro de la cuba de fusión. Así mismo se presenta la posible distribución de las partículas de 


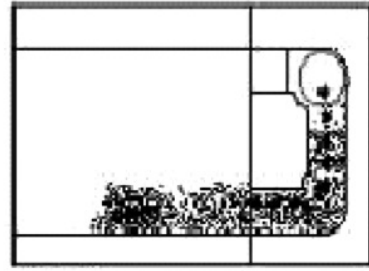

a) $5.4 \mathrm{~s}$

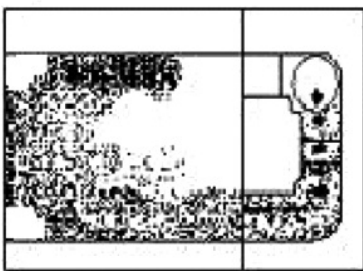

d) $21.6 \mathrm{~s}$

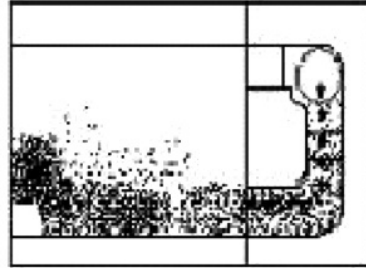

b) $10.8 \mathrm{~s}$

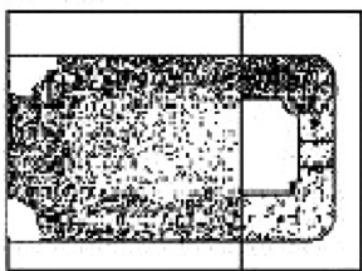

e) $27 \mathrm{~s}$

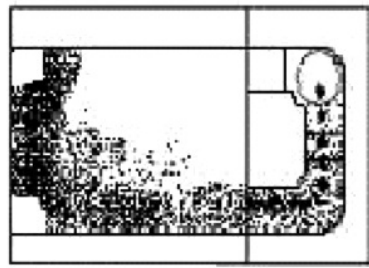

c) $16.2 \mathrm{~s}$

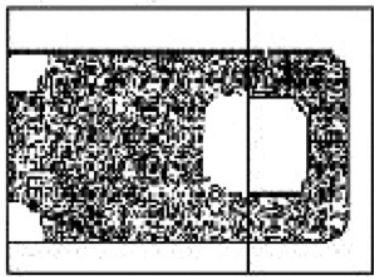

f) $32.4 \mathrm{~s}$

Figura 5. Secuencia esquemática de la trayectoria y distribución de las partículas de baquelita transportadas por el flujo de fluido, para el IT rotando a $351 \mathrm{rpm}$, para una relación $h i / h$.

Figure 5. Schematic sequence of the trajectories and distribution of solid particles transported by the fluid, when using a IT impeller rotating at $351 \mathrm{rpm}$, for a hi / h ratio.

baquelita transportadas por el mismo flujo. Se presume que las partículas siguen la misma trayectoria que el líquido y por lo tanto la distribución es como se muestra en las figuras $5 \mathrm{a}$ ), b) y c). Como se puede observar en las figuras $5 \mathrm{~d}$ ) -f), las se van integrando, gradualmente, al resto del volumen de la cámara, desde los bordes hacia el centro.

\subsection{Resultados de la cuba de fusión con modificaciones}

De acuerdo a lo mencionado anteriormente, los tiempos de circulación y mezclado pueden ser relativamente largos para las condiciones ensayadas hasta ahora, por lo que se propuso realizar algunas modificaciones al modelo físico con el fin de disminuir los tiempos de circulación y mezclado y, así, agilizar la recirculación del líquido por todo el sistema. En el conjunto de las figuras 6 y 7 se aprecian los comportamientos exhibidos por el IT y I3P, respectivamente, para el modelo modificado.

$\mathrm{Al}$ inicio del proceso se observan algunas variaciones en la absorbancia asociadas al avance del frente del colorante y a la formación de regiones segregadas en la cuba de fusión. Además, el efecto de las modificaciones parece ser significativo, en ambos casos, ya que los tiempos de circulación se ven afectados con las modificaciones hechas al modelo físico. En el caso del IT, durante las primeras etapas del proceso de mezclado se presentan una serie de fluctuaciones importantes asociadas al frente de colorante y a las nuevas zonas estancadas, para las dos velocidades estudiadas. En el caso del impulsor I3P, las reducciones son por casi la mitad del tiempo indicado para el modelo sin modificaciones. Solo en uno de los casos, para una relación de altura de $h i / h=0,25$ a una velocidad de $255 \mathrm{rpm}$, el I3P presenta un comportamiento más estable. Para este tipo de impulsor, la velocidad de agitación tiene un efecto importante en la reducción del tiempo de mezclado ya que al incrementarse la velocidad de agitación se incrementa la recirculación, permitiendo que el proceso sea más estable y eficiente. De acuerdo con lo descrito anteriormente, no cabe duda que las modificaciones en el modelo físico alteraron el patrón de flujo, teniendo el líquido una mejor recirculación, disminuyendo por consiguiente los tiempos de circulación y de mezclado. En el caso del I3P en el modelo modificado se tienen tiempos de circulación cortos, independientemente de la posición del impulsor debido a que el líquido recorre una distancia menor, de aproximadamente $260 \mathrm{~cm}$.

En las figuras 8 a) y b) se presenta, en forma esquemática, la distribución del fluido en todo el sistema y las nuevas zonas de estancamiento formadas en la cuba de fusión para las modificaciones realizadas. Como puede observarse, la distribución del líquido en la cuba de fusión sigue el patrón marcado por las modificaciones, rompiendo el flujo hacia el centro de la cuba y dividiendo el patrón que se formaba antes de las modificaciones, donde el líquido seguía las paredes hasta el fondo de la cuba. El flujo entre las compuertas adquiere mayor velocidad obstruyendo la formación de partes estancadas en esta zona, 

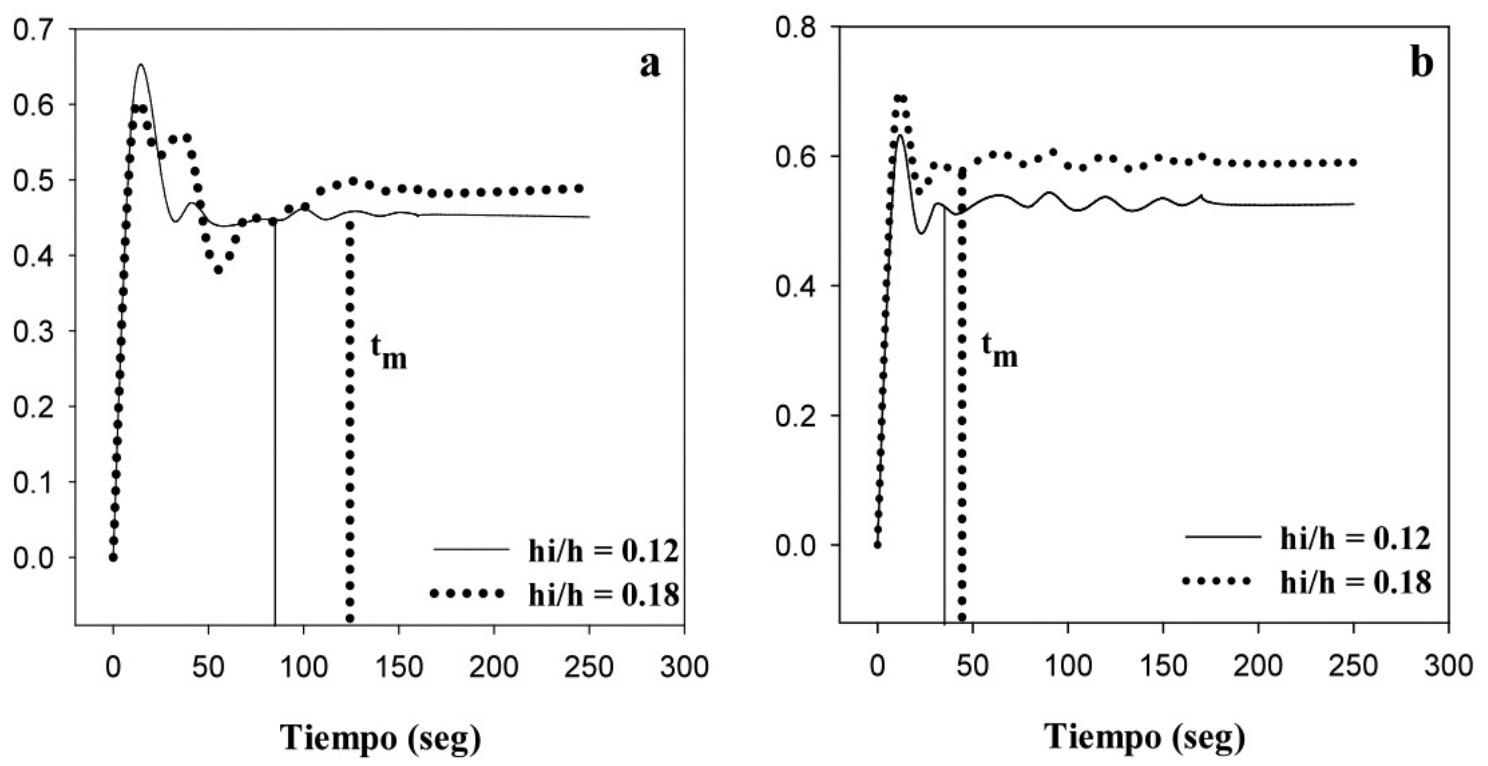

Figura 6. Relación entre la absorbancia y tiempo de mezclado del IT, a) 200 rpm; b) 351 rpm, en el modelo con modificaciones.

Figure 6. Relationship between absorbance and mixing time for an IT, a) rotating at $200 \mathrm{rpm}$; b) rotating at $351 \mathrm{rpm}$, after geometrical modifications to the physical model.

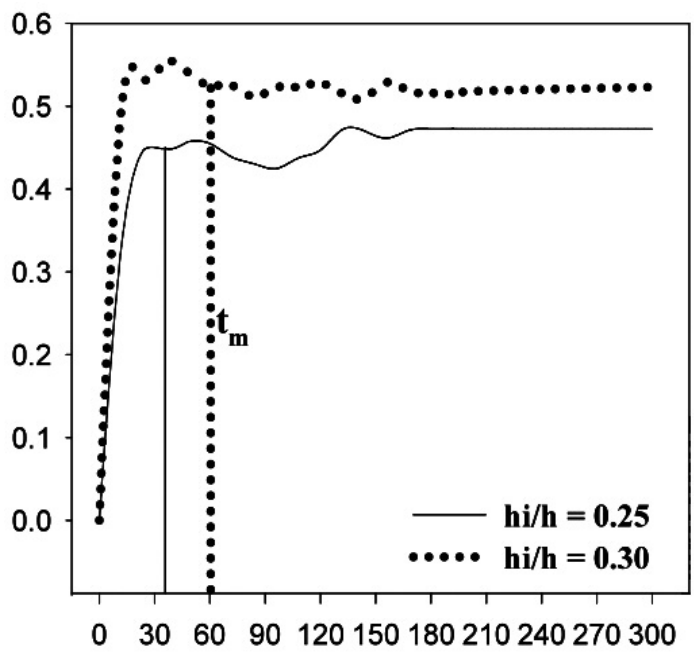

Tiempo (seg)

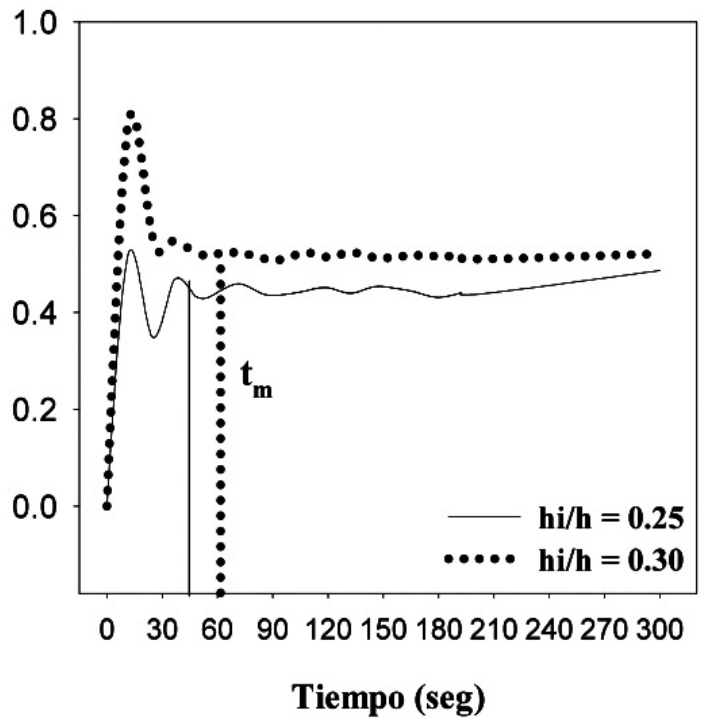

Figura 7. Relación entre la absorbancia y tiempo de mezclado del $/ 3 P$, a) 255 rpm; b) 292 rpm, en el modelo con modificaciones.

Figure 7. Relationship between absorbance and mixing time for an I3B, a) rotating at $255 \mathrm{rpm}$; b) rotating at $292 \mathrm{rpm}$, after geometrical modifications to the physical model.

por lo que, ahora, más volumen del líquido en la cuba principal tiene una mejor recirculación y un retorno más acelerado hacia la compuerta que comunica a la cuba de agitación. Sin embargo, se detectó la presencia de dos pequeñas zonas estancadas, las cuales se ilustran en la figura 8 b). Por su tamaño, estas zonas no interfieren de manera abrupta en el proceso de recirculación y mezclado. En otras palabras, las modificaciones intermedias representan un cinturón que "estrecha" el patrón de flujo existente dividiéndolo a manera de un "8". El patrón de flujo que sigue la baquelita es el mismo que sigue el líquido, por 


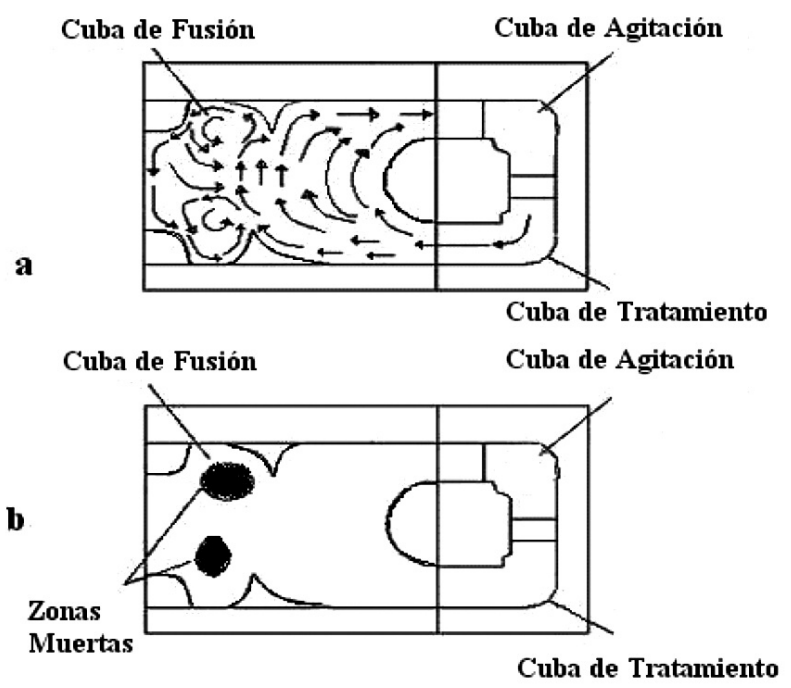

Figura 8. a) Representación esquemática del perfil de flujo de fluido después de las modificaciones geométricas de la cuba de fusión; b) localización de las nuevas zonas estancadas.

Figure 8. a) Schematic representation of the fluid flow profile after geometrical modifications to the melting chamber; b) location of the new stagnant zones detected.

lo tanto, el conjunto líquido-partículas se comportan de manera semejante a través de todo el sistema. La tabla VI muestra la reducción promedio de los tiempos de mezclado para las velocidades indicadas para un conjunto de pruebas realizadas con el I3P.

\subsection{Modelación matemática, cuba de fusión}

El comportamiento del líquido obtenido en las simulaciones correspondientes a la cuba de fusión se mues- tra en la figura 9, donde se observa que las zonas de mayor velocidad dentro de la cuba corresponden a la entrada y salida de las compuertas de comunicación, la zona intermedia corresponde a la zona de mayor recirculación (la mitad de la cuba), mientras que en las esquinas del sistema (parte posterior) la velocidad del fluido es cero, lo que demuestra la formación de zonas estancadas en esta parte del sistema. La cuba de fusión presenta dos zonas de movimiento lento en las esquinas de la cuba, al igual que en la zona entre las compuertas de comunicación. Las zonas de mayor movimiento están en la compuesta de entrada, que corresponde a la cuba de tratamiento, y la compuerta que comunica con la cuba de agitación.

En cuanto a la trayectoria de partículas, la figura 10 presenta una serie de imágenes (el color rojo corresponde a la mayor concentración de partículas y el azul a la mínima) donde se muestra como se lleva a cabo la dispersión de las partículas de baquelita agregadas en el sistema y el tiempo que tardan en incorporarse en la cuba de agitación sin modificaciones. En dichas imágenes se puede observar que conforme el conjunto líquido-partículas avanza por el sistema, sigue el mismo patrón de flujo presentado por el líquido ya que al inicio, cuando ingresa a la cuba de fusión, el frente partículas-líquido se guía por la pared hasta alcanzar la parte posterior de la cuba (en aprox. 100 s), para después girar y seguir por la pared opuesta hasta completar el ciclo (en aprox. 300 s). Al igual que en los casos anteriores, la zona más lenta ocurre entre las compuertas de comunicación donde las partículas tardan en incorporarse al sistema. De hecho, es la zona donde no se alcanza una completa homogenización. En este caso, las esquinas de la cuba de fusión se convirtieron en zonas de mezclado aislado, permitiendo que las partículas se incorporaran lentamente al flujo y siguieran el patrón del mismo. La relación entre la densidad del aluminio líquido y la arena sílice, con un tamaño de

Tabla VI. Tiempos de mezclado medido antes y después de las modificaciones, cuando se usa el I3P

Table. VI. Mixing time measured before and after the geometrical modifications, when using the I3P

\begin{tabular}{cccc}
\hline $\begin{array}{c}\text { Velocidad } \\
\text { (rpm) }\end{array}$ & $\begin{array}{c}\text { Antes de las } \\
\text { modificaciones }\end{array}$ & $\begin{array}{c}\text { Después de las } \\
\text { modificaciones }\end{array}$ & $\begin{array}{c}\text { Reducción en el } \\
\text { tiempo de mezclado }\end{array}$ \\
\hline \multirow{2}{*}{255} & 84 & 36 & $57 \%$ \\
& 96 & 48 & $50 \%$ \\
292 & 72 & 48 & $34 \%$ \\
& 84 & 66 & $21 \%$ \\
\hline
\end{tabular}




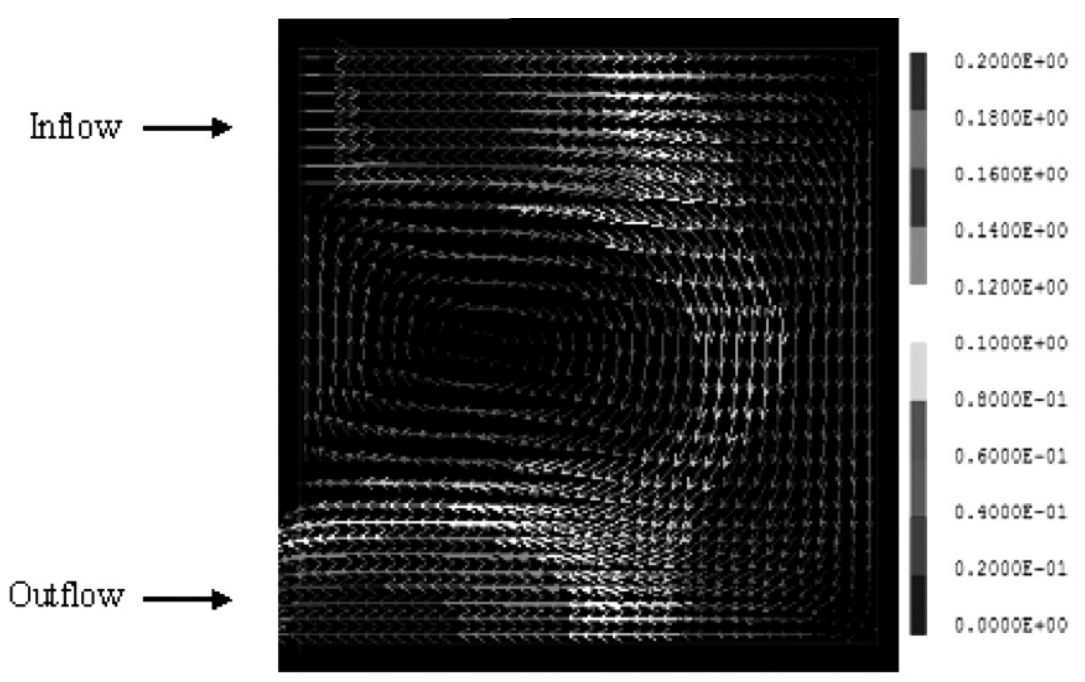

Figura 9. Patrón de flujo en la cuba de fusión, para el $/ 3 P$ a $255 \mathrm{rpm}$, para la relación $h i / h=0,25$.

Figure 9. Numerically predicted fluid flow pattern and velocity profile in the melting chamber, for I3B an impeller rotating at $255 \mathrm{rpm}$, for a hi/ $\mathrm{h}=0.25$ ratio.
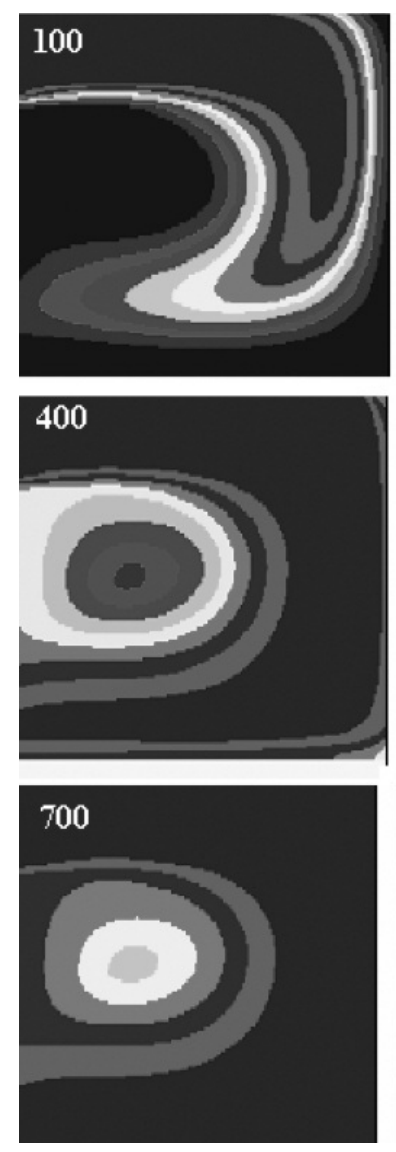
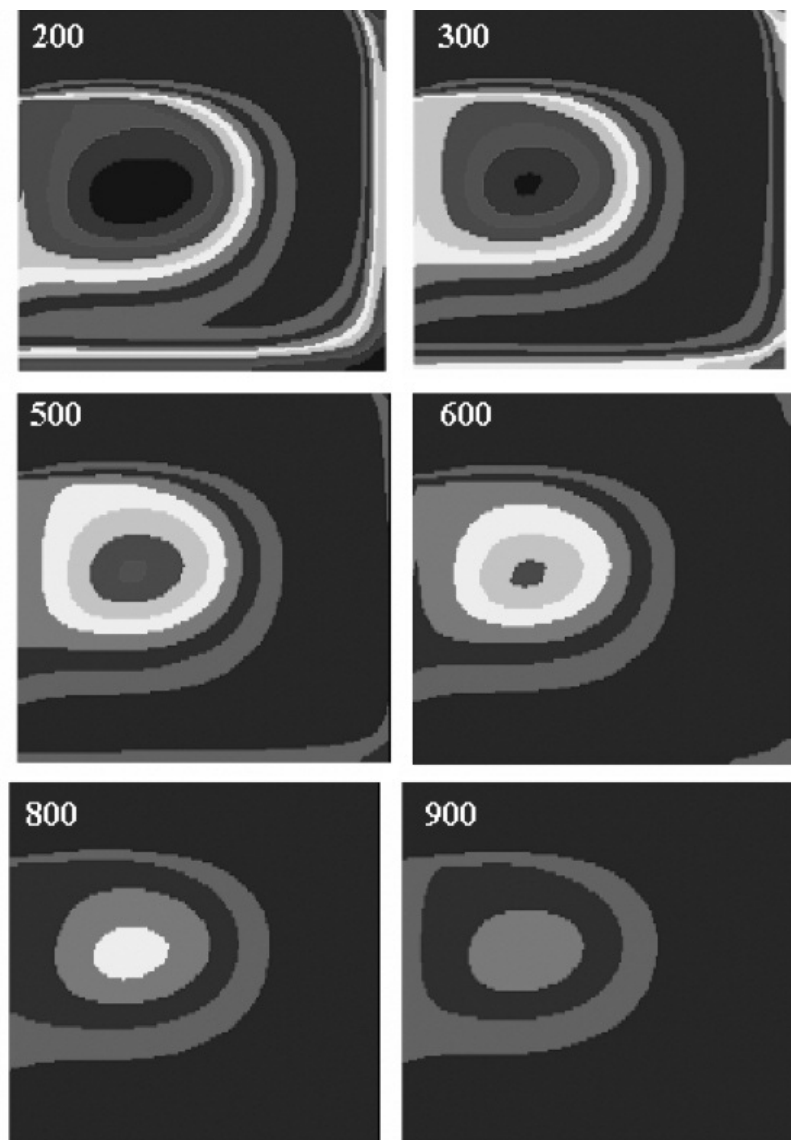

Figura 10. Secuencia de la distribución de las partículas de baquelita transportadas por el flujo de fluido, dada en segundos, para un $/ 3 P$ a $255 \mathrm{rpm}$, para una relación $h i / h=0,25$.

Figure 10. Sequence of the trajectories and distribution of solid particles transported by the fluid flow, predicted for an I3B impeller rotating at $255 \mathrm{rpm}$, for a hi/ $\mathrm{h}=0.25$ ratio, as a function of time (in seconds). 
partícula que oscila entre $210-106 \mu \mathrm{m},\left(\gamma_{p}\right)$. Por otro lado, si la densidad de la baquelita ${ }^{[22]}$ es $1,27 \mathrm{~kg} / \mathrm{m}^{3}$, entonces, la relación entre la densidad del agua y la baquelita, $\gamma_{m}=0,79$, estará cercana a la presentada por el metal y la arena (en sí, la concentración fue baja, ya que no se puede usar la cantidad de arena requerida por lo impracticable que sería en el modelo físico). Por lo tanto, se utilizaron partículas de baquelita para obtener los tiempos de circulación debido a que su comportamiento se asemeja más al de las partículas de arena. Por el contrario, los tiempos de circulación determinados con el colorante fueron más cortos a causa de que éste se diluye y se desplaza con mayor ligereza dentro del seno del agua. No obstante, el colorante fue más útil para medir tiempos de mezclado en el agua que de manera análoga, vendría siendo el tiempo de homogenizado en el horno.

\subsection{Modelación matemática en la cuba de fusión modificada}

Se realizaron algunas simulaciones numéricas con el sistema modificado, con los resultados mostrados en la figura 11. Cabe señalar que a la malla se le agregaron nuevos nodos con el fin de simular las trampas colocadas al sistema, observándose que el patrón de flujo del fluido es como se planteó en el modelo físico, es decir, un patrón de flujo en forma de "ocho", con una sección de flujo continuo que corresponde a la sección donde se encuentran las trampas. Aquí, se divide el patrón de flujo en dos secciones: una, de mayor movimiento, localizada a la mitad de la cuba y una sección de flujo lento, en la parte posterior de la cuba, donde el líquido experimenta la velocidad más baja y lentamente se incorpora al resto del sistema.

\section{ESCALAMIENTO A NIVEL INDUSTRIAL}

El escalamiento de los resultados obtenidos durante la modelación física y numérica hacia un proceso industrial como el horno de reverbero implica múltiples fenómenos: físicos, mecánicos, térmicos y químicos. Lamentablemente, el hecho de mejorar las condiciones de mezclado del fluido y encontrar la forma de obtener los tiempos de circulación y mezclado más cortos no constituyen el éxito sobre la operación de dicho horno como unidad de refinación, específicamente la disminución de magnesio del baño metálico mediante el empleo de arena sílice. Esto, tiene que ver con las consideraciones cinéticas y termodinámicas que conlleva la reacción química involucrada ya que una vez formada la capa de productos de reacción sobre las partículas de sílice, el proceso es controlado por una etapa de difusión del magnesio disuelto hacia el centro de la partícula, etapa que es muy lenta. De ahí, la diferencia en tiempos de homogenizado en el modelo fisco y tiempos de remoción de magnesio a escala industrial. Ya que los criterios de similitud satisfechos en la experimentación indican que el patrón del flujo de fluido es similar en ambos sistemas, se puede tomar como

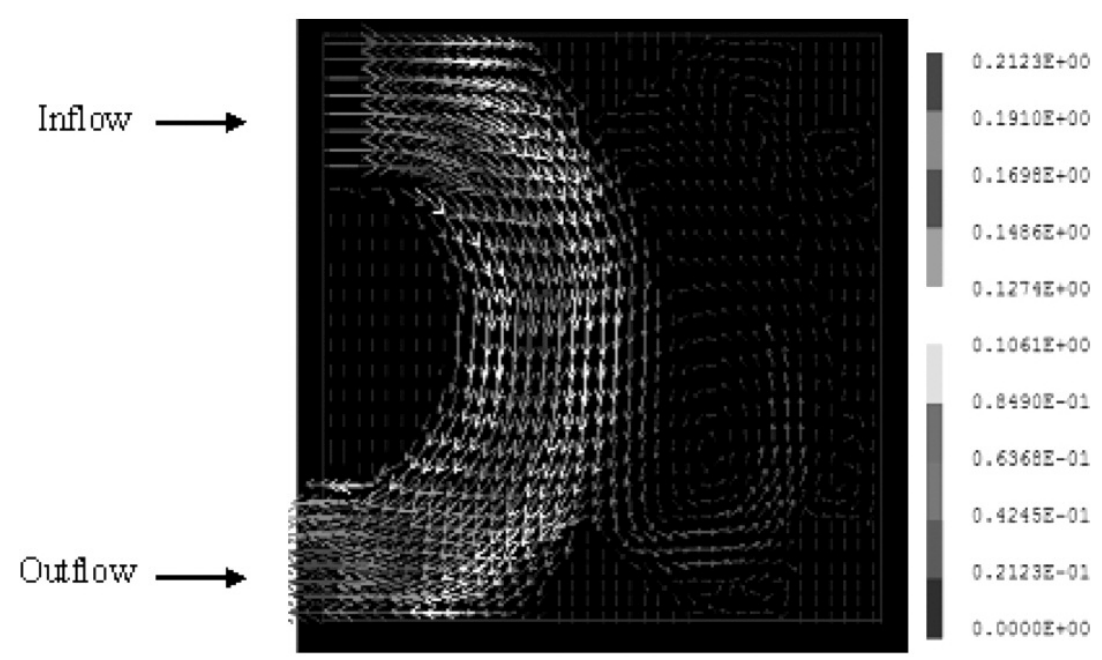

Figura 11. Patrón de flujo en la cuba de fusión con modificaciones, para el $13 P$ a $255 \mathrm{rpm}$, para una relación $h i / h=0,25$.

Figure 11. Predicted sequence of the fluid flow for the geometrically modified melting chamber, when using an I3B impeller rotating at $255 \mathrm{rpm}$, for a hi/ $\mathrm{h}=0.25$ ratio. 
confiables las aproximaciones hechas a través de las ecuaciones utilizadas para escalar las variables de respuesta de un sistema a otro. El escalamiento se realizó utilizando la ecuación utilizada por Mazundar ${ }^{[16]}$ según la cual, los tiempos de homogenización del baño metálico son 1,68 veces mayores que los tiempos de mezclado en el modelo físico, escala 1:2, dada por:

$$
\left(t_{m}\right)_{m}=\left(t_{m}\right)_{p} \lambda^{3 / 4}
$$

donde, $\left(t_{m}\right)_{m}$ es el tiempo de mezclado en el modelo físico, en segundos y $\left(t_{m}\right)_{p}$ es el tiempo de mezclado en el prototipo industrial, también en segundos. $\lambda$ es el factor de escala geométrica. Considerando, para este caso, un factor de escala dado por $\lambda=0,5$, se obtiene la siguiente expresión:

$$
\left(t_{m}\right)_{p}=1,68\left(t_{m}\right)_{m}
$$

La tabla VII muestra los resultados de escalamiento del modelo físico, a nivel industrial, para los tipos de impulsores utilizados girando a diferentes velocidades, para una relación $h i / h=0,25$.

Tabla VII. Resultados del escalamiento de los $\left(t_{m}\right)_{p}$ del HRDC a partir de los $\left(t_{m}\right)_{m}$ del modelo físico, para una relación hi/h $=0,25$

Table VII. Results of scaling to the $\left(t_{m}\right)_{p}$ of $H R D C$ from the results of physical model, for the $\mathrm{hi} / \mathrm{h}=0,25$ ratio

\section{Modelo físico actual utilizando solamente un impulsor}

\begin{tabular}{crrrr}
\hline & \multicolumn{1}{c}{ I2P } & \multicolumn{2}{c}{ I3P } \\
& $\boldsymbol{N}_{\boldsymbol{m}}(\mathbf{r p m})$ & \multicolumn{2}{c}{$\boldsymbol{N}_{\boldsymbol{m}}(\mathbf{r p m})$} \\
\hline modelo, s & 255 & 292 & 255 & 292 \\
HRDC, s & 108 & 84 & 72 & 108 \\
\hline \multicolumn{4}{c}{ Modelo físico modificado utilizando } \\
solamente un impulsor
\end{tabular}

Cabe mencionar que no hay forma de mantener todos los criterios de escalamiento constantes entre dos sistemas. En el presente trabajo se cumplió con varios de estos criterios como la similitud geométrica, el número de Froude para agitadores y, de forma muy aproximada, se conservó la misma potencia por unidad de volumen y relaciones muy parecidas entre la densidad del líquido y la del sólido. Lo anterior, permite establecer que las condiciones fluidinámicas logradas en el modelo físico se pueden lograr en el prototipo, por lo tanto, se lograrían tiempos de residencia largos para que las partículas de sílice reaccionaran completamente, así como una buena dispersión de estas por todo el sistema, esperando alcanzar tiempos de eliminación de magnesio más cortos. En otras palabras, el presente estudio ha permitido identificar, de manera óptima, un rango de valores que deben tener las variables del proceso a nivel industrial, así como la descripción de zonas estancadas en el sistema, pudiendo establecer con estos resultados que el proceso de disminución de magnesio en un horno de reverbero de doble cámara puede ser eficiente bajo estas condiciones de operación. En este sentido, la figura 12, muestra resultados obtenidos a nivel industrial durante la inyección de sílice en la cámara de agitación de un horno de reverbero de 5 t de capacidad, antes y después de las modificaciones geométricas y mecánicas sugeridas, específicamente, el rediseño del horno y el aumento de la velocidad de rotación

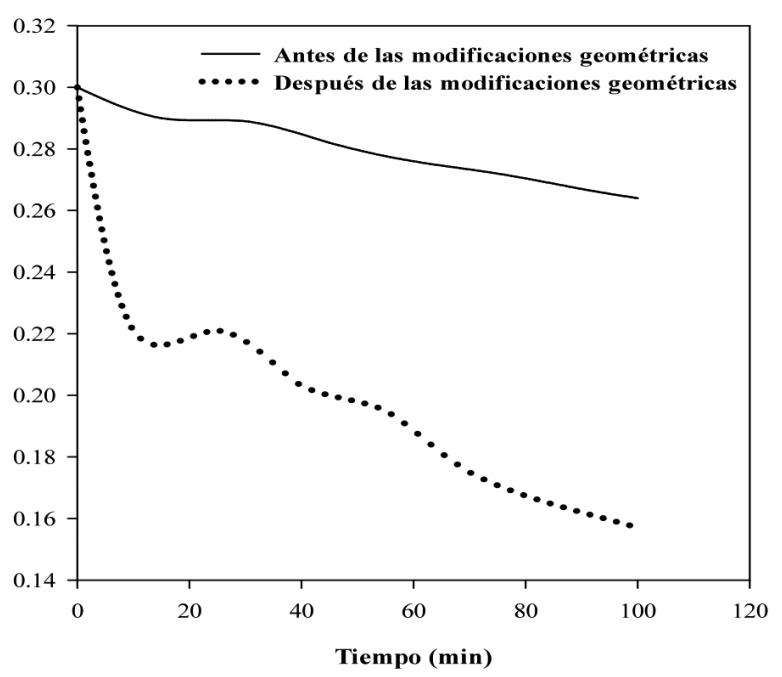

Figura 12. Resultados de la velocidad de remoción de $\mathrm{Mg}$ a nivel industrial usando el $/ 3 P$, para una relación $h i / h=0,25$.

Figure 12. Magnesium removal rate results corresponding to the use of an I3B rotating at 180 rpm, for a hi/ $\mathrm{h}=0.25$ ratio. 
de un impulsor I3P, desde 180 hasta $255 \mathrm{rpm}$. Como puede verse, aumentó significativamente la tasa de disminución de magnesio y, por consiguiente, la eficiencia de reacción. Como se determinó a partir de este trabajo, la notoria mejoría en los tiempos de circulación y de mezclado a través de modificaciones geométricas y mecánicas, sugieren la posibilidad de solicitar formas más redondeadas de las principales cámaras del horno de reverbero, ya que el diseño de este tipo de hornos es completamente rectangular. De hecho, muy recientemente, se están ofreciendo tecnologías ${ }^{[17 \text { y } 18]}$ que consideran el horno de reverbero como un verdadero horno circular, reclamando al mismo tiempo una mayor eficiencia, tanto en los procesos de fusión como en los de combustión.

\section{CONCLUSIONES}

De acuerdo a los resultados obtenidos en este trabajo, la simulación física y matemática del flujo de fluidos y trayectorias de partículas sólidas podría usarse para simular las condiciones operacionales de un horno de reverbero usado para la fusión y refinación de aluminio secundario. Los resultados muestran que la recirculación del flujo es afectada por varios aspectos, principalmente la geometría del la cuba de fusión, ya que este tipo de reactores son completamente rectangulares y, por ello, dificulta la circulación del líquido en el sistema. De igual importancia es el efecto que presenta el tipo de impulsor y la posición de este con respecto a la compuerta de comunicación entre las cubas de agitación y tratamiento. Debido a esto, se realizaron modificaciones geométricas de la cuba de agitación y la cuba de fusión para tener una mejor recirculación en todo el sistema y romper las zonas estancadas, modificaciones que permitieron tener una mejor distribución de las partículas, prolongando el tiempo de residencia en el baño metálico. El impulsor que obtuvo el mejor rendimiento en las dos características necesarias como bombeo y mezclado es el I3P. Finalmente, se puede decir que de acuerdo a las velocidades del fluido y, por tanto, a las de las partículas sólidas, el horno de reverbero se puede usar como una unidad de refinación ya que la reacción de eliminación de magnesio tiene el tiempo suficiente para que las partículas de $\mathrm{SiO}_{2}$ agregadas reaccionen completamente. Esto se demostró durante las pruebas a nivel industrial, donde las reacciones de eliminación fueron más eficientes después de efectuar los cambios de diseño de las cubas e incrementar la velocidad de agitación del impulsor.

\section{Agradecimientos}

Los autores desean agradecer al Ing. Jesús Velázquez Rodríguez, Director General de Fundición J.V. S.A. de C.V., por las facilidades experimentales otorgadas. Así mismo, agradecen al Consejo Nacional de Ciencia y Tecnología de México, por el apoyo financiero otorgado, Proyecto ECO-2006-C01-52822.

\section{REFERENCIAS}

[1] C. Martínez, Tesis de Maestría, CINVESTAV, 1998.

[2] S. L. Yeoh, G Papadakis y M. Yianneskis, Chem. Eng. Sci. 60 (2005) 2.293-2.302.

[3] C. Galleti, A.P. Aglianti y M. Yianneskis, Chem. Eng. Sci. 60 (2005) 2.317-2.331.

[4] L Nikiforaki, G Montante, K.C. Lee, M. Yianneskis, Chem. Eng. Sci. 58 (2003) 2.937-2.949.

[5] Y. Murakami, T. Hirose, T. Yamato, H. Fujiwara y M. Ohshima, J. Chem. Eng. Jpn. 13 (1980) 318-323.

[6] T. Nomura, T. Uchida y K. Takahashi, J. Chem. Eng. Jpn. 30 (1997) 875-879.

[7] W.G. Yao, H. Soto, K Takahashi y K. Koyama, Chem. Eng. Sci. 52 (1997) 807-814.

[8] A.A. Barresi. Chem. Eng. Sci. 53 (1998), pp. 3.031-3.040.

[9] A. Flores, C. Martinez, J.C. Escobedo, J.A. Toscano y A. Puente, Light Metals. (1999) 711-718.

[10] J.C. Escobedo, J.F. Hernandez, S. Escobedo, A. Flores y D.A. Cortes, Rev. Met. Madrid 39 (2003) 172-182.

[11] F. Barajas, Tesis de Maestría en Ing. Metalúrgica, Centro de Investigación y Estudios Avanzados del IPN, 2004.

[12] D.J. Lamberto, M.M. Alvarez y F.J. Muzzio, Chem. Eng. Sci. 54 (1999) 919-942.

[13] B.E. Launder y D.B. Spalding, Comput. Method.Appl. Mech.Eng. 3 (1974) 269-289.

[14] R. I. Guthrie, Engineering in Process Metallurgy, Oxford University Press, EE.UU., 1992, pp. 174-175, 178.

[15] N.B. Reyes, Tesis Maestría en Ing. Metalúrgica, Centro de Investigación y Estudios Avanzados del IPN, Ramos Arizpe Coah. 2006.

[16] D. Mazundar, Tesis Doctoral, McGill Univ., Montreal, Canadá, 1985.

[17] Patent US2005/0035503 A1.

[18] Patent US2008/0023891 A1. 